\title{
Sparse BRDF Approximation using Compressive Sensing
}

\author{
Benoit Zupancic \\ INRIA - Grenoble University
}

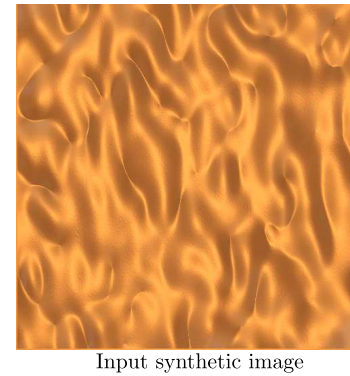

(MERL gold paint)
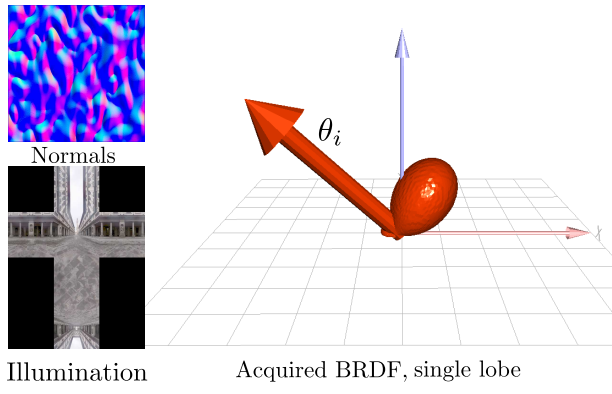

Acquired BRDF, single lobe

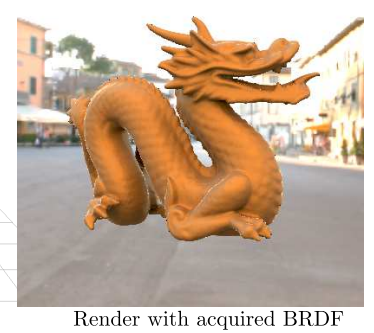

(Pisa environment map)

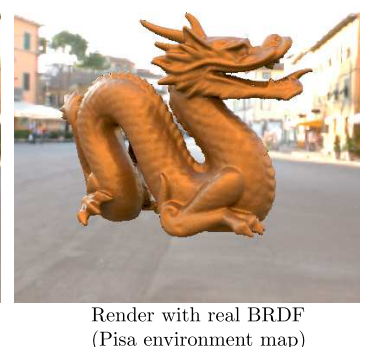

(Pisa environment map)

Figure 1: We show that it is possible to compute a sparse approximation of a BRDF from an image (a) with known lighting (b) and normals (c), using compressive sensing. The acquired BRDF (d) can further be used to generate valid images in a different illumination/geometry setup. This is a very promising step toward the acquisition of material properties from a single image.

\section{Introduction}

BRDF acquisition is a tedious operation, since it requires measuring $4 \mathrm{D}$ data. On one side of the spectrum lie explicit methods, which perform many measurements to potentially produce very accurate reflectance data after interpolation [Matusik et al. 2003]. These methods are generic but practically difficult to setup and produce high volume data. On the other side, acquisition methods based on parametric models implicitly reduce the infinite dimensionality of the BRDF space to the number of parameters, allowing acquisition with few samples. However, parametric methods require non linear optimization. They become unstable when the number of parameters is large, with no guaranty that a given parametric model can ever fit particular measurements.

We experiment a new acquisition method where the measurement of the BRDF is performed from a single image, knowing the normals and illumination. To tackle such a severely underconstrained problem, we express the BRDF in a high dimensional basis, and perform the reconstruction using compressive sensing, looking for the most sparse solution to the linear problem of fitting the measurement image. Doing so, we leverage the coherency between the measured pixels, while keeping the high dimension of the space the BRDF is searched into.

This work is a very first attempt at reconstructing BRDFs using compressive sensing. In Fig 1 we used a synthetic input image, for the sake of checking the feasibility of the recovery algorithm, in the particular case of an isotropic spatially constant BRDFs. The possibility to extend our theory to non spatially varying and anisotropic BRDFs is currently under investigation. We would like to orient our work toward BRDF acquisition with consumer hardware. In particular, our preliminary results indicate that compressive sensing could achieve an very accurate acquisition with additional input, such as a video of a static object under probed lighting.

\section{Sparse BRDF optimization}

The compressive sensing theory has shown that sparse fitting of linear constraints can be achieved by solving

$$
\mathbf{x}=\underset{\mathbf{y}}{\operatorname{argmin}}\|A \mathbf{y}-\mathbf{b}\|_{L_{2}}+\lambda\|\mathbf{y}\|_{L_{1}} .
$$

In our case, $\mathbf{x}$ is the vector of coefficients of the BRDF in a high dimensional basis, $A$ is a matrix measuring the linear dependency between the unknown BRDF and the measured image - also expressed in a linear basis. $\mathbf{b}$ is the particular measurement we want to reproduce, meaning the coefficients of the image in this basis, and $\lambda$ is a sparsity factor.

We use the SpaRSA algorithm for fitting which is a variant of Iterated Hard Thresholding [Wright et al. 2009]. Starting from an arbitrary coefficient vector $\mathbf{x}_{0}$, we iterate:

$\mathbf{x}_{n+1}=S\left(\mathbf{x}_{n}+\frac{1}{\alpha_{n}} A^{T}\left(\mathbf{b}-A \mathbf{x}_{n}\right), \frac{\lambda}{\alpha_{n}}\right) \quad$ with $\quad \alpha_{n}=\frac{\left\|A \mathbf{x}_{n}\right\|_{2}^{2}}{\left\|\mathbf{x}_{n}\right\|_{2}^{2}}$

In this equation, $S$ is the soft-threshold operator, that removes the smaller coefficients, essentially making it sparse, and defined by $S(a, b)=\operatorname{sign}(a) \max (0,|a|-b)$.

The BRDF is expressed in a Fourier basis: for a given input direction $\theta_{i}$ the output lobe is expressed into spherical harmonics, and the spherical harmonics coefficients are decomposed into cosines as a function of $\theta_{i}$ :

$$
\rho\left(\theta_{i}, \theta_{o}, \phi_{o}\right)=\sum_{-l \leq m \leq l} \sum_{k=0}^{N} \lambda_{k, l, m} \cos \left(\frac{k \theta_{i}}{N}\right) y_{l}^{m}\left(\theta_{o}, \phi_{o}\right)
$$

This work raises - and partially answers - some interesting questions: a single image can be used to acquire a BRDF, as long as both the cone of normals and the range of illumination frequencies are large enough. We show such an example in Figure 1. Forcing the captured BRDF to have a physical nature (e.g. reciprocity) can be achieved with an appropriate parameterization. Works by E. Candès give the number of measurements that ensure the convergence is almost certain, which gives a clue of the amount of data that is necessary. convergence is usually very fast. Most of computation cost relies in the precomputation of the matrix $A$.

\section{References}

Matusik, W., Pfister, H., Brand, M., and McMillan, L. 2003. A data-driven reflectance model. In ACM SIGGRAPH 2003 Papers, ACM, New York, NY, USA, SIGGRAPH '03, 759-769.

Wright, S. J., Nowak, R. D., And Figueiredo, M. A. T. 2009. Sparse reconstruction by separable approximation. Trans. Sig. Proc. 57, 7 (July), 2479-2493. 